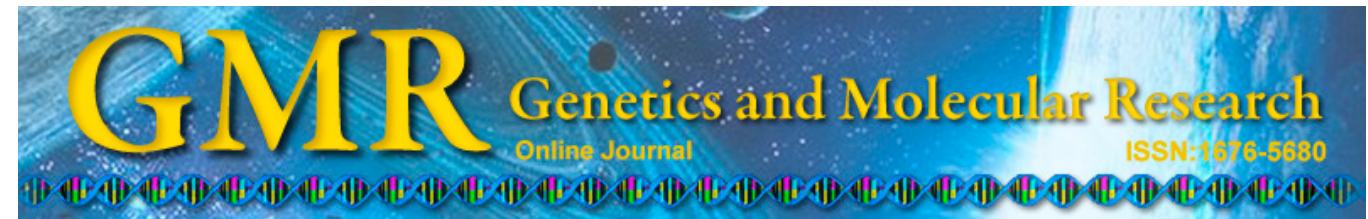

\title{
Genetic diversity in intraspecific hybrid populations of Eucommia ulmoides Oliver evaluated from ISSR and SRAP molecular marker analysis
}

\author{
J. Yu ${ }^{1}$, Y. Wang ${ }^{2}$, M. Ru ${ }^{2}$, L. Peng ${ }^{1}$ and Z.S. Liang ${ }^{1,2}$ \\ ${ }^{1}$ College of Life Sciences, Northwest A\&F University, Yangling, China \\ ${ }^{2}$ The Center of Soil and Water Conservation and Ecoenvironmental Research, \\ Chinese Academy of Sciences, Yangling, China \\ Corresponding author: Z.S. Liang \\ E-mail: liangzs@ms.iswc.ac.cn
}

Genet. Mol. Res. 14 (3): 7417-7425 (2015)

Received September 3, 2014

Accepted March 9, 2015

Published July 3, 2015

DOI http://dx.doi.org/10.4238/2015.July.3.17

\begin{abstract}
Eucommia ulmoides Oliver, the only extant species of Eucommiaceae, is a second-category state-protected endangered plant in China. Evaluation of genetic diversity among some intraspecific hybrid populations of E. ulmoides Oliver is vital for breeding programs and further conservation of this rare species. We studied the genetic diversity of 130 accessions from 13 E. ulmoides intraspecific hybrid populations using inter-simple sequence related (ISSR) and sequencerelated amplified polymorphism (SRAP) markers. Of the 100 ISSR primers and 100 SRAP primer combinations screened, eight ISSRs and eight SRAPs were used to evaluate the level of polymorphism and discriminating capacity. A total number of 65 bands were amplified using eight ISSR primers, in which 50 bands $(76.9 \%)$ were polymorphic, with an average of 8.1 polymorphic fragments per primer. Alternatively, another 244 bands were observed using eight SRAP primer combinations, and $163(66.8 \%)$ of them were polymorphic,
\end{abstract}


with an average of 30.5 polymorphic fragments per primer. The unweighted pair-group method (UPGMA) analysis showed that these 13 populations could be classified into three groups by the ISSR marker and two groups by the SRAP marker. Principal coordinate analysis using SRAP was completely identical to the UPGMA-based clustering, although this was partly confirmed by the results of UPGMA cluster analysis using the ISSR marker. This study provides insights into the genetic background of $E$. ulmoides intraspecific hybrids. The progenies of the variations "Huazhong-3", "big fruit", "Yanci", and "smooth bark" present high genetic diversity and offer great potential for E. ulmoides breeding and conservation.

Key words: Eucommia ulmoides Oliver; Genetic diversity; ISSR; SRAP; Intraspecific hybrids

\section{INTRODUCTION}

Eucommia ulmoides Oliver ('Du-zhong' in Chinese) is a living fossil plant; it is a tertiary relict species that is only found in China (Takeno et al., 2008). The bark of E. ulmoides has been used in traditional Chinese medicine for more than 2000 years. It possesses the pharmacological effects of nourishing the liver and kidney, strengthening the muscles and bones, preventing miscarriage, as well as lowering blood pressure and blood sugar (Hsieh and Yen, 2000; Deyama et al., 2001; Kwan et al., 2003; Committee CP, 2010). Gutta-percha, which is an important industrial gum (Yan, 1989), is found in the E. ulmoides leaves, bark, and seed shell (Tangpakdee et al., 1997). As a second-category state-protected endangered plant in China, E. ulmoides has an endangered status in the wild, but it is widely cultivated in China, Russia, Japan, and other countries for economic benefits.

E. ulmoides is a deciduous tree that represents a significant germplasm source with different morphological variations: rough and smooth bark; short and winding branch; big and small fruit; long petiole, small, large, giant, and purple-red leaf (Du, 1997). Conventional breeding of E. ulmoides has mainly focused on the selection of promising plants from existing natural populations. Breeding goals for $E$. ulmoides mainly target high yield, resistance to diseases, high content of medical compounds, and gutta-percha. Qinzhong No. 1-4 (Zhang et al., 2004) and Huazhong No. 1-5 (Du et al., 1994) were selected for these desirable characteristics. These selected plants were vegetatively propagated and released as clones ( $\mathrm{Li}$ et al., 2014). However, biased asexual reproduction might not be appropriate for the conservation of the genetic variation because this material is not suitable for long-term storage as seeds.

Previously, studies of the genetic diversity of E. ulmoides were based largely on natural or artificial populations throughout the main distribution in China (Wu et al., 2011; Yao et al., 2012; Zhang et al., 2013). There is a limited number of molecular studies that have focused on the genetic diversity of intraspecific genotypes and their hybrid progenies. Meanwhile, cross hybridization between diverse elite parental clones to create progenies with maximum genetic variations is important for improving breeding processes (Perera et al., 2012). Among various molecular tools for genetic analysis, inter-simple sequence repeat (ISSR) is a powerful tool, because its application does not need any prior information about the target sequences in the genome (Wang et al., 2012). Sequence-related amplified polymorphism (SRAP) has been 
shown to be more informative than other PCR-based markers for detecting genetic diversity (Budak et al., 2004). Here, ISSR and SRAP markers were used to investigate the genetic diversity of 130 hybrid progenies that were obtained from 13 E. ulmoides female clones for the first time. The objective of this study was to identify a potentially more genetically diverse germplasm source for germplasm conservation and especially future breeding progress.

\section{MATERIAL AND METHODS}

\section{Plant materials and genomic DNA extraction}

The list of 13 studied E. ulmoides intraspecific hybrids and their origins are provided in Table 1. Seeds were collected and planted in the medicinal botanical garden at Northwest A\&F University, China. Young, mature, unfolded leaves of seedlings were collected for DNA extraction. Total genomic DNA was extracted from about $100 \mathrm{mg}$ fresh leaves using the KangWei NuClean PlantGen DNA Kit. The DNA was then suspended in TE buffer. DNA concentration and quality were evaluated by electrophoresis on $1.0 \%$ agarose gel. DNA was stored at $-20^{\circ} \mathrm{C}$.

Table 1. Details of the germplasm sources of Eucommia ulmoides and sample number.

\begin{tabular}{llcl}
\hline Population & Origin & Sample No. & Female parent \\
\hline HZ2-LY & Luoyang, Henan Province & 10 & Huazhong-2 \\
HZ3-LY & & 10 & Huazhong-3 \\
HZ4-LY & Sanmenxia, Henan Province & 10 & Huazhong-4 \\
HZ2-SMX & & 10 & Huazhong-2 \\
HZ4-SMX & & 10 & Huazhong-4 \\
HL-SMX & & 10 & Giant leaf \\
BL-SMX & & 10 & Large leaf \\
SL-SMX & & 10 & Small leaf \\
BF-SMX & & 10 & Big fruit \\
GL-SMX & 10 & Short branch \\
YC-SMX & & 10 & Yanci \\
SB-SMX & & 10 & Smooth bark \\
SB-HZ & Hanzhong, Shannxi Province & 10 & Smooth bark \\
\hline
\end{tabular}

\section{PCR amplification}

One hundred ISSR primers (Isshiki et al., 2008) (University of British Columbia, Canada) were used for initial screening. Of these, eight primers (Table 2) generated reproducible and highly polymorphic bands. Amplification reactions were performed in $20-\mu \mathrm{L}$ volumes containing $2.0 \mu \mathrm{L} 10 \mathrm{X}$ PCR buffer, $2.0 \mathrm{mM} \mathrm{MgCl}, 200 \mathrm{mM}$ dNTPs, $1 \mathrm{mM}$ ISSR primers, $1 \mathrm{U}$ Taq DNA polymerase (KangWei, China), and approximately $10 \mathrm{ng}$ template DNA. PCR amplification was performed on the $\mathrm{AB}$ Applied Biosystems thermocycler (Gene Company Limited, USA) under the following PCR program: 5 min of denaturing at $94^{\circ} \mathrm{C}, 40$ cycles of three steps: $30 \mathrm{~s}$ of denaturing at $94^{\circ} \mathrm{C}, 30 \mathrm{~s}$ of annealing at $58^{\circ} \mathrm{C}$, and $1 \mathrm{~min}$ of elongation at $72^{\circ} \mathrm{C}$, with a final elongation step of $10 \mathrm{~min}$ at $72^{\circ} \mathrm{C}$. The PCR products were separated by electrophoresis on $1.5 \%$ agarose gel using $1 \mathrm{X}$ TBE buffer, $\mathrm{pH} 8.0$, at room temperature and a 5000-bp ladder with standard molecular size (KangWei). The gel was visualized with ethidium bromide staining. 
SRAP analysis was carried out according to previously established protocols described by Li and Quiros (2001). Of these 100 SRAP primer pairs, eight primer combinations that consistently produced clear and diverse amplified bands were selected (Table 2). The PCR mixtures ( $20 \mu \mathrm{L}$ total volume) contained $2.0 \mu \mathrm{L} 10 \mathrm{X}$ PCR buffer, $2.0 \mathrm{mM} \mathrm{MgCl}, 200 \mathrm{mM}$ dNTPs, $0.5 \mathrm{mM}$ forward primer, $0.5 \mathrm{mM}$ reverse primer, $1 \mathrm{U}$ Taq DNA polymerase (KangWei), and approximately $20 \mathrm{ng}$ template DNA. PCR amplification was performed on the AB Applied Biosystems (Gene Company Limited), with the following PCR program: 5 min of denaturing at $94^{\circ} \mathrm{C}, 5$ cycles of three steps: $1 \mathrm{~min}$ of denaturing at $94^{\circ} \mathrm{C}, 1 \mathrm{~min}$ of annealing at $35^{\circ} \mathrm{C}$, and $2 \mathrm{~min}$ of elongation at $72^{\circ} \mathrm{C}$. In the following 35 cycles, the annealing temperature was increased to $50^{\circ} \mathrm{C}$, with a final elongation step of $8 \mathrm{~min}$ at $72^{\circ} \mathrm{C}$. The PCR products were separated on 6\% non-denaturing polyacrylamide gel, and SRAP bands were stained using silver sequence DNA staining reagents and a 5000-bp ladder molecular size standard (KangWei).

\section{Data analysis}

Amplified bands were scored either as present (1) or absent (0) as well as scored for a binary data matrix with polymorphic and reproducible bands. The matrix was then used for the following analysis: the number of effective loci, the percentage of polymorphic loci (PPB), the effective number of alleles, observed number of alleles, Shannon's information index (I) (Lewontin, 1995), and Nei' gene diversity (H) (Nei, 1973) were obtained by the package POPGENE 3.2 version 1.31 software (Yeh et al., 1997). To further examine the genetic relationships among accessions, cluster analysis based on the genetic similarity matrix was performed with the UPGMA (unweighted pair group method with arithmetic mean) method (Sneath and Sokal), using the SHAN function of the NTSYS-pc version 2.1 (Rohlf, 2000). A principal coordinates analysis (PCoA) was performed using the GenAlEx version 6.5 software (Peakall and Smouse, 2012).

\section{RESULTS}

\section{ISSR and SRAP analysis}

We surveyed 130 E. ulmoides accessions that represented 13 intraspecific hybrid populations using eight ISSR primers (Table 2).

\begin{tabular}{|c|c|c|c|c|}
\hline ISSR primer sequences & Sequences $\left(5^{\prime} \rightarrow 3^{\prime}\right)$ & SRAP primer combinations & Forward primer sequences & Reverse primer sequences \\
\hline UBC825 & $(\mathrm{AC})_{8} \mathrm{~T}$ & Me2Em8 & TGAGTCCAAACCGGAGC & GACTGCGTACGAATTAGC \\
\hline UBC826 & $(\mathrm{AC})_{8}^{8} \mathrm{C}$ & Me5Em8 & TGAGTCCAAACCGGAAG & GACTGCGTACGAATTAGC \\
\hline UBC828 & $(\mathrm{TG})_{8}^{8} \mathrm{~A}$ & Me7Em4 & TGAGTCCAAACCGGTCC & GACTGCGTACGAATTTGA \\
\hline UBC848 & $(\mathrm{CA})_{8}^{8} \mathrm{RG}$ & Me8Em4 & TGAGTCCAAACCGGTGC & GACTGCGTACGAATTTGA \\
\hline UBC851 & $(\mathrm{GT})_{8}^{8} \mathrm{YG}$ & Me8Em9 & TGAGTCCAAACCGGTGC & GACTGCGTACGAATTACG \\
\hline UBC856 & $(\mathrm{AC})_{8}^{8} \mathrm{YT}$ & Me9Em5 & TGAGTCCAAACCGGACA & GACTGCGTACGAATTAAC \\
\hline UBC857 & $(\mathrm{AC})_{8} \mathrm{TA}$ & Me10Em5 & TGAGTCCAAACCGGAGA & GACTGCGTACGAATTAAC \\
\hline UBC881 & $\left(\mathrm{GGGTG}_{3}\right.$ & Me10Em9 & TGAGTCCAAACCGGAGA & GACTGCGTACGAATTACG \\
\hline
\end{tabular}

A total of 65 bands were identified, of which 50 were polymorphic (76.9\%), with a minimum of five (UBC851) and a maximum of 12 (UBC848) bands per primer (Table 3). Representative banding patterns, which were detected by the primer UBC826, are shown in Figure 
1. The size of amplified fragments ranged from 0.2 to $2 \mathrm{~kb}$, with an average of 8.1 fragments per primer. The percentage of polymorphic bands that were produced by each primer ranged from $40 \%$ (UBC851) to $91.7 \%$ (UBC848).

\begin{tabular}{|c|c|c|c|c|c|c|c|}
\hline ISSR primer & Band generated & $\mathrm{N}$ & PPB (\%) & SRAP primer combinations & Band generated & $\mathrm{N}$ & PPB (\%) \\
\hline UBC825 & 9 & 8 & 88.9 & $\mathrm{Me} 2 \mathrm{Em} 8$ & 15 & 7 & 46.7 \\
\hline UBC 826 & 8 & 4 & 50.0 & Me5Em8 & 46 & 31 & 67.4 \\
\hline UBC 828 & 7 & 6 & 85.7 & Me7Em4 & 23 & 14 & 60.9 \\
\hline UBC848 & 12 & 11 & 91.7 & Me8Em4 & 27 & 14 & 51.9 \\
\hline UBC 851 & 5 & 2 & 40.0 & Me8Em9 & 18 & 11 & 61.1 \\
\hline UBC856 & 8 & 7 & 87.5 & Me9Em5 & 36 & 25 & 69.4 \\
\hline UBC857 & 9 & 7 & 77.8 & Me10Em5 & 35 & 27 & 77.1 \\
\hline UBC881 & 7 & 5 & 71.4 & Me10Em9 & 44 & 34 & 77.3 \\
\hline Total & 65 & 50 & 76.9 & Total & 244 & 163 & 66.8 \\
\hline Average & 8.1 & 6.3 & & Average & 30.5 & 20.4 & \\
\hline
\end{tabular}

$\mathrm{N}=$ number of polymorphic bands; $\mathrm{PPB}=$ percentage of polymorphic bands.

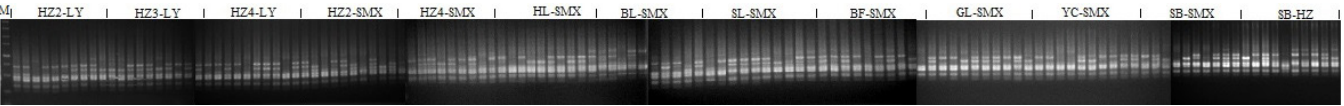

Figure 1. Intrapopulation polymorphism revealed by ISSR markers produced with primer UBC826.

The same samples were then analyzed with eight SRAP primer combinations (Table 2). They yielded a total of 244 fragments, of which 163 were polymorphic $(66.8 \%)$, with a minimum of 15 (Me2Em8) and a maximum of 44 (Me10Em9) bands per primer combination (Table 3). Representative banding patterns, which were detected by the primer combination Me10Em5, are shown in Figure 2. The size of amplified fragments ranged from 0.1 to $5 \mathrm{~kb}$, with an average of 30.5 fragments per primer. The percentage of polymorphic bands that were produced by each primer pair ranged from 46.7\% (Me2Em8) to 77.3\% (Me10Em9).

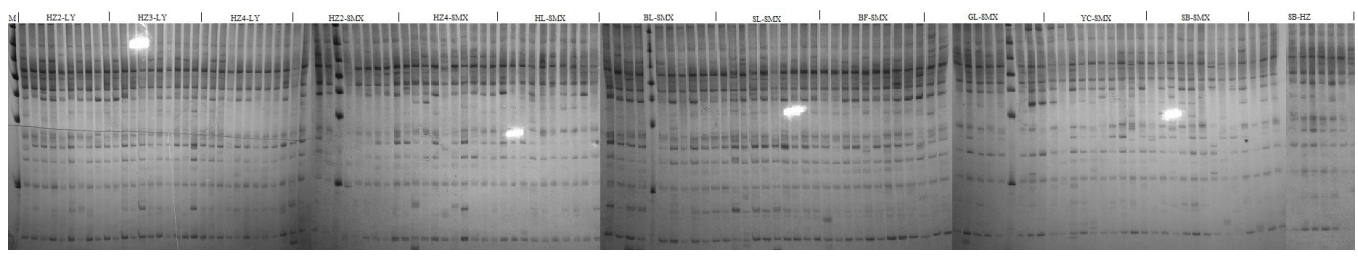

Figure 2. Intrapopulation polymorphism revealed by SRAP markers produced with primer combination Me10Em5.

\section{Level of genetic variation}

Based on our ISSR analysis, the PPB ranged from 20.0 to $52.3 \%$, with a mean of $40.0 \%$ (Table 4). The values of genetic diversity, as estimated by $\mathrm{H}$, varied from 0.080 to 0.211 . I ranged from 0.117 to 0.308 . Our SRAP analysis indicated that the PPB ranged from 31.8 to $54.1 \%$, with a mean of $44.1 \%$ (Table 4 ). The values of genetic diversity as estimated $\mathrm{H}$ varied from 0.122 to 0.192 . I ranged from 0.180 to 0.286 . Both ISSR and SRAP analysis showed that HZ3-LY, BF-SMX, YC-SMX, SB-SMX, and SB-HZ had high genetic diversity, while BL-SMX and HL-SMX had relatively low genetic diversity. 
Table 4. Genetic diversity of Eucommia ulmoides intraspecific hybrids revealed by combined ISSR and SRAP markers.

\begin{tabular}{|c|c|c|c|c|c|c|c|c|c|c|}
\hline \multirow[t]{2}{*}{ Pop } & \multicolumn{5}{|c|}{ ISSR } & \multicolumn{5}{|c|}{ SRAP } \\
\hline & $N_{\mathrm{A}}$ & $N_{\mathrm{E}}$ & $\mathrm{H}$ & I & PPB (\%) & $N_{\mathrm{A}}$ & $N_{\mathrm{E}}$ & $\mathrm{H}$ & I & PPB (\%) \\
\hline HZ2-LY & 1.385 & 1.302 & 0.165 & 0.237 & 38.5 & 1.459 & 1.292 & 0.172 & 0.256 & 45.9 \\
\hline HZ2-SMX & 1.385 & 1.302 & 0.165 & 0.237 & 38.5 & 1.364 & 1.256 & 0.145 & 0.212 & 36.4 \\
\hline HZ3-LY & 1.446 & 1.272 & 0.160 & 0.240 & 44.6 & 1.541 & 1.305 & 0.184 & 0.278 & 54.1 \\
\hline HZ4-LY & 1.323 & 1.227 & 0.129 & 0.189 & 32.3 & 1.409 & 1.262 & 0.154 & 0.229 & 40.9 \\
\hline HZ4-SMX & 1.339 & 1.210 & 0.125 & 0.187 & 33.9 & 1.376 & 1.256 & 0.147 & 0.216 & 37.6 \\
\hline HL-SMX & 1.292 & 1.190 & 0.111 & 0.165 & 29.2 & 1.318 & 1.210 & 0.122 & 0.180 & 31.8 \\
\hline BL-SMX & 1.200 & 1.142 & 0.080 & 0.117 & 20.0 & 1.388 & 1.255 & 0.148 & 0.220 & 38.8 \\
\hline SL-SMX & 1.385 & 1.286 & 0.159 & 0.231 & 38.5 & 1.409 & 1.273 & 0.158 & 0.233 & 40.9 \\
\hline BF-SMX & 1.523 & 1.346 & 0.196 & 0.289 & 52.3 & 1.521 & 1.323 & 0.189 & 0.282 & 52.1 \\
\hline GL-SMX & 1.400 & 1.276 & 0.155 & 0.228 & 40.0 & 1.417 & 1.259 & 0.151 & 0.226 & 41.7 \\
\hline YC-SMX & 1.492 & 1.319 & 0.183 & 0.272 & 49.2 & 1.488 & 1.288 & 0.170 & 0.255 & 48.8 \\
\hline SB-SMX & 1.508 & 1.345 & 0.193 & 0.283 & 50.8 & 1.508 & 1.328 & 0.190 & 0.281 & 50.8 \\
\hline SB-HZ & 1.523 & 1.375 & 0.211 & 0.308 & 52.3 & 1.533 & 1.333 & 0.192 & 0.286 & 53.3 \\
\hline Mean & 1.400 & 1.276 & 0.156 & 0.229 & 40.0 & 1.441 & 1.280 & 0.163 & 0.286 & 44.1 \\
\hline Total & 1.754 & 1.457 & 0.264 & 0.394 & & 1.690 & 1.392 & 0.234 & 0.352 & \\
\hline
\end{tabular}

$N_{\mathrm{A}}=$ observed number of alleles; $N_{\mathrm{E}}=$ effective number of alleles; $\mathrm{H}=$ Nei's gene diversity; I $=$ Shannon's information index; $\mathrm{PPB}=$ percentage of polymorphic loci.

\section{Cluster analysis}

A dendrogram was plotted using an UPGMA analysis that was derived from the ISSR profile data and is shown in Figure 3a. The 13 populations were divided into four clusters at a similarity coefficient of 0.87 . Cluster 1 comprised population HZ2-LY and BL-SMX. Cluster 2 contained eight populations (HZ2-SMX, SB-SMX, SB-HZ, HZ4-LY, HZ3-LY, HZ4-SMX, SL-SMX, and GL-SMX). Populations BF-SMX and YC-SMX were separated into cluster 3. Alternatively, the progeny of one population, HL-SMX, had the biggest leaf variation and formed cluster 4, which was an out-group for the rest of the clusters. The SRAP-based dendrogram showed three clusters with a GS coefficient of 0.91 (Figure 3b). Three populations (HZ2LY, GL-SMX, and YC-SMX) formed cluster 1. Cluster 2 comprised population HZ2-SMX, HZ3-LY, SB-SMX, SB-HZ, HZ4-LY, BF-SMX, BL-SMX, and SL-SMX. Cluster 3 consisted of populations HZ4-SMX and HL-SMX.
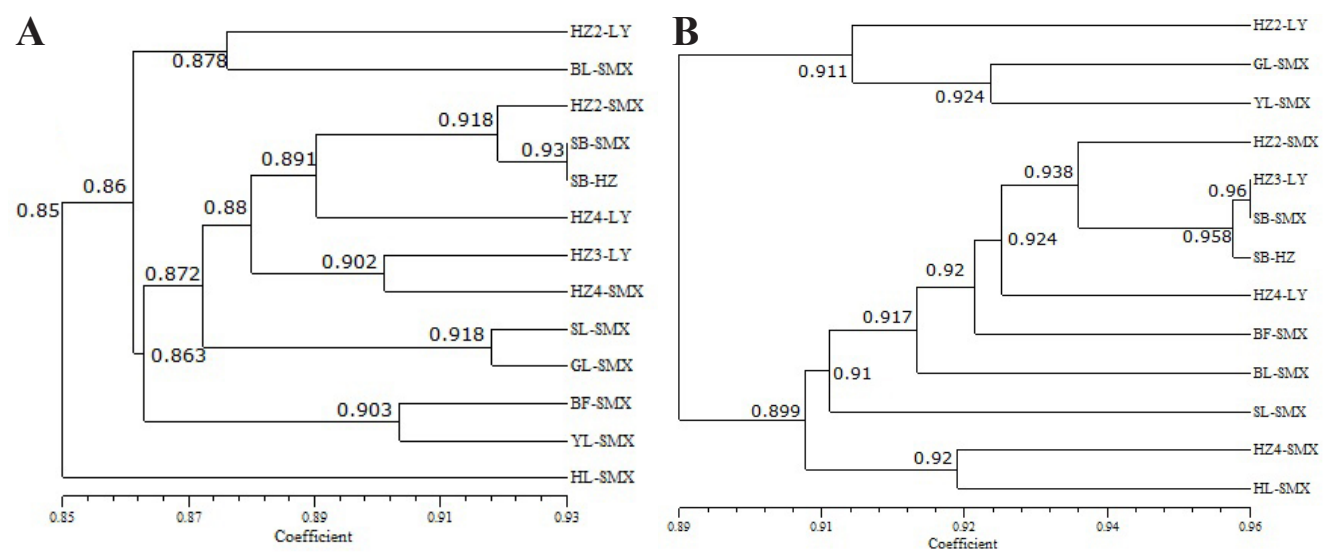

Figure 3. UPGMA dendrogram for the Eucommia ulmoides intraspecific hybrid populations based on Nei's genetic distance, as revealed using A. ISSR markers and B. SRAP markers. 
Results from the PCoA that was based on genetic similarity matrices revealed that the first two principal components accounted for 52.2 and $62.6 \%$ of the total variation using ISSR and SRAP markers, respectively. UPGMA analysis and PCoA analysis using the SRAP marker were identical. However, PCoA results were partly confirmed by the results of UPGMA cluster analysis revealed by the ISSR marker (Figure 4).

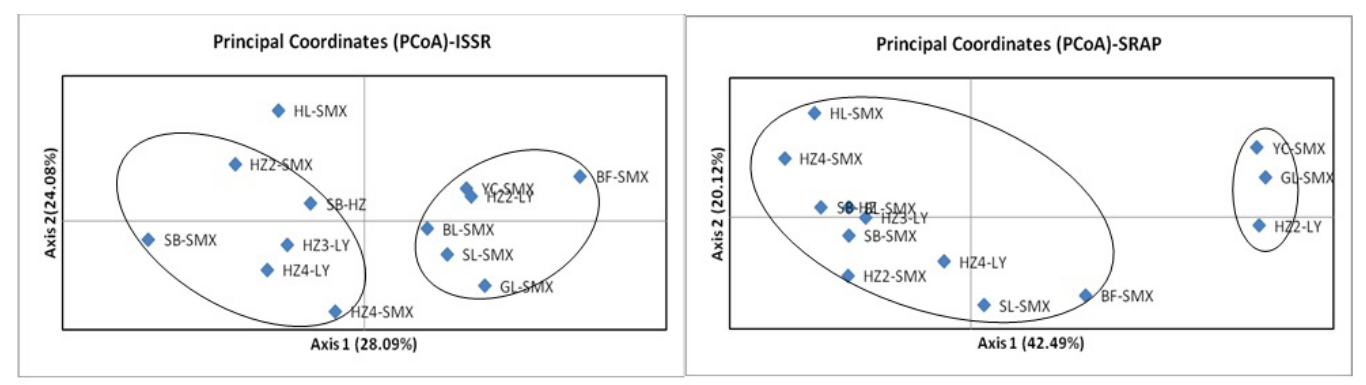

Figure 4. Principal coordinate analysis (PCoA) revealed by ISSR and SRAP markers using the genetic similarity matrix for the Eucommia ulmoides intraspecific hybrid populations.

\section{DISCUSSION}

Using genetically distant germplasm as parents to generate better performing hybrids is important for breeding programs (Perera et al., 2012). In traditional E. ulmoides breeding, morphological, physical, and chemical characters were used to analyze the varieties. Moreover, broad molecular markers were used in genetic diversity analyses (Zhang et al., 2013) and QTL mapping (Li et al., 2014; Wang et al., 2014) of E. ulmoides in recent years, because they were not affected by the environment, the physiological state of the plant, and other factors. Hence, we selected 13 E. ulmoides hybrid populations for the breeding program and germplasm conservation based on ISSR and SRAP.

In this study, eight ISSRs and eight pieces of SRAP marker systems were used in characterizing the genetic diversity of 13 E. ulmoides hybrid populations. Here, we found both efficiency and utility of these two markers. These markers revealed very high genetic diversity in the hybrids. ISSR revealed a higher polymorphic band number $(76.9 \%)$ compared with SRAP (66.8\%). However, the SRAP marker amplified a higher number of bands (30.5 per primer combinations on average) than ISSR (8.1 per primer on average).

The main objective of this study was to identify high genetic diversity of the E. ulmoides intraspecific hybrids for breeding programs and future germplasm conservation. Prior studies indicated that E. ulmoides exhibited high genetic diversity at the species level but a relatively lower genetic diversity at the population level (Yao et al., 2012; Zhang et al., 2013). As a tertiary relict species, E. ulmoides accumulated a large amount of genetic variation during their long evolutionary history, which also provides a valuable resource for higher genetic diversity. A diverse collection of E. ulmoides germplasm may provide an opportunity to broaden the genetic base and a boost to the current breeding program.

Based on our ISSR and SRAP analysis, the results showed that HZ3-LY, BF-SMX, YC-SMX, SB-SMX, and SB-HZ had high genetic diversity. The clones "Huazhong-2", "Huazhong-3", "big fruit", and "Yanci" were selected for their ability to breed high-quality 
seeds. The common characteristic of "Huazhong-3" (Du et al., 1994) and "Yanci" is that their seeds mature earlier than other female clones, and the seeds have a higher germination capability. In addition, the "big fruit" seeds are widely used in industrialized utilization of extraction of aucubin and $\alpha$-linolenic acid for the higher kernel ratio ( $\mathrm{Du}, 1997)$. However, there are no correlations between the characteristics of female clones and higher genetic diversity of hybrids. It is necessary to investigate the genetic diversity of female clones themselves. Interestingly, improved varieties that had better seed productivity and quality also had a large proportion of genetic diversity.

Our results also revealed that SB-SMX and SB-HZ had high genetic diversity. Combined ISSR and SRAP markers as well as UPGMA dendrogram and PCoA analysis (Figures 1 and 2) both showed that the "smooth bark" variation from two geographic regions was closely grouped together. Unlike other female clones, this variation was found both in male and female trees. They seemed have higher genetic diversity and better stability. The "smooth bark" variety was characterized by its higher medical compound production. The stable high genetic diversity and medical benefits gave this variation a priority for germplasm collection and improved variations breeding of $E$. ulmoides species.

Moreover, the genetic diversities of BL-SMX and HL-SMX were relatively low. The "giant" and "large" leaves are too big for pollen dispersal to occur. The lower genetic diversity of the "giant" and "large" leaves could be the second reason. Biased asexual reproduction for desirable characteristics might result in genetic erosion and an alarmingly narrow genetic base. In addition, the UPGMA dendrogram and PCoA analysis that were obtained from ISSR data all indicated that "giant leaf" hybrids were distinct from other groups. These two clones are useful for breeding programs rather than genetic conservation.

Our current study provides new insight into information of the genetic backgrounds of E. ulmoides intraspecific hybrids. The ISSR and SRAP polymorphisms highlighted that abundant genetic variability existing within hybrids, indicating that these progenies offer great potential for E. ulmoides improvement and conservation.

\section{ACKNOWLEDGMENTS}

Research supported by Seeds and Seedlings Quality Standard of Du-zhong (\#2012ZX09304006), which is the sub-task of Major Chinese National Science and Technology Special Funds "Quality Standard Platform of Planting (Breeding) Seeds and Seedlings of Traditional Chinese Medicine".

\section{REFERENCES}

Budak H, Shearman RC, Parmaksiz I, Gaussoin RE, et al. (2004). Molecular characterization of Buffalograss germplasm using sequence-related amplified polymorphism markers. Theor. Appl. Genet. 108: 328-334.

Committee CP (2010). Chinese Pharmacopoeia (I). China Medica Science Press, Beijing. 161-162.

Deyama T, Nishibe S and Nakazawa Y (2001). Constituents and pharmacological effects of Eucommia and Siberian ginseng. Acta Pharmacol. Sin. 22: 1057-1070.

Du H (1997). Variant cultivar types of Eucommia in China. Econ. For. Res. 15: 34-36.

Du H, Zhang Z, Liu B and Du L (1994). Selection and breeding of five excellent clones of Eucommia ulmoides. J. Northwest Forestr. Coll. 4: 27-31.

Hsieh CL and Yen GC (2000). Antioxidant actions of du-zhong (Eucommia ulmoides Oliv.) toward oxidative damage in biomolecules. Life Sci. 66: 1387-1400. 
Isshiki S, Iwata N and Khan MMR (2008). ISSR variations in eggplant (Solanum melongena L.) and related Solanum species. Sci. Hort. 117: 186-190.

Kwan CY, Chen CX, Deyama T and Nishibe S (2003). Endothelium-dependent vasorelaxant effects of the aqueous extracts of the Eucommia ulmoides Oliv. leaf and bark: implications on their antihypertensive action. Vascul. Pharmacol. 40: 229-235.

Lewontin RC (1995). The apportionment of human diversity. Evol. Biol. 6: 381-398.

Li G and Quiros CF (2001). Sequence-related amplified polymorphism (SRAP), a new marker system based on a simple PCR reaction: its application to mapping and gene tagging in Brassica. Theor. Appl. Genet. 103: 455-461.

Li Y, Wang D, Li Z, Wei J, et al. (2014). A molecular genetic linkage map of Eucommia ulmoides and quantitative trait loci (QTL) analysis for growth traits. Int. J. Mol. Sci. 15: 2053-2074.

Nei M (1973). Analysis of gene diversity in subdivided populations. Proc. Natl. Acad. Sci. U. S. A. 70: 3321-3323.

Peakall R and Smouse PE (2012). GenAlEx 6.5: genetic analysis in Excel. Population genetic software for teaching and research - an update. Bioinformatics 28: 2537-2539.

Perera MF, Arias ME, Costilla D, Luque AC, et al. (2012). Genetic diversity assessment and genotype identification in sugarcane based on DNA markers and morphological traits. Euphytica 3: 491-510.

Rohlf F (2000). NTSYS-pc, Numerical Taxonomy Multivariate Analysis System. Version 2.1. Exceter Software, New York.

Sneath P and Sokal R (1973). Numerical Taxonomy. WF Freeman and Co., San Francisco.

Takeno S, Bamba T, Nakazawa Y, Fukusaki E, et al. (2008). Quantification of trans-1, 4-polyisoprene in Eucommia ulmoides by Fourier transform infrared spectroscopy and pyrolysis-gas chromatography/mass spectrometry. $J$. Biosci. Bioeng. 105: 355-359.

Tangpakdee J, Tanaka Y, Shiba K-I, Kawahara S, et al. (1997). Structure and biosynthesis of trans-polyisoprene from Eucommia ulmoides. Phytochemistry 45: 75-80.

Wang D, Li Y, Li L, Wei Y, et al. (2014). The first genetic linkage map of Eucommia ulmoides. J. Genet. 93:13-20.

Wang X-M, Hou X-Q, Zhang Y-Q, Yang R, et al. (2012). Genetic diversity of the endemic and medicinally important plant Rheum officinale as revealed by inter-simple sequence repeat (ISSR) markers. Int. J. Mol. Sci. 13: 3900-3915.

Wu MQ, Chen SL, Wang MX and Yan SZ (2011). An analysis of the genetic diversity and genetic structure of Eucommia ulmoides using inter-simple sequence repeat (ISSR) markers. Afr. J. Biotechnol. 10: 19505-19513.

Yan R (1989). An age-old and young natural polymer - gutta-percha. Chin. Polymer Bull. 2: 39-44.

Yao X, Deng J and Huang H (2012). Genetic diversity in Eucommia ulmoides (Eucommiaceae), an endangered traditional Chinese medicinal plant. Conserv. Genet. 13: 1499-1507.

Yeh FC, Yang R-C, Boyle TB, Ye Z, et al. (1997). POPGENE, The User-Friendly Shareware for Population Genetic Analysis. Molecular Biology and Biotechnology Centre, University of Alberta, Edmonton.

Zhang B, Zhang K, Zhang T, Su Y, et al. (2004). Researches on the selection and breeding of superior species of Qinzhong 1-4. J. Northwest Forestr. Coll. 19: 18-20.

Zhang J, Xing C, Tian H and Yao X (2013). Microsatellite genetic variation in the Chinese endemic Eucommia ulmoides (Eucommiaceae): implications for conservation. Bot. J. Linn. Soc. 173: 775-785. 